\title{
Searches for SUSY in leptons+jets+MET final states
}

\author{
Natascha HOERMANN* ${ }^{*}$ \\ Institute of High Energy Physics, Austrian Academy of Science, Vienna, Austria \\ E-mail: hoermann@hephy . oeaw.ac.at
}

If supersymmetry would manifest itself at a low mass scale it might be found already in the early phase of the LHC operation. Generic signatures for supersymmetry in pp-collisions consist of high jet multiplicity, large missing transverse energy (MET) as well as leptons in the final state. The presence of charged leptons makes these signature more robust and therefore facilitates their application in early data-taking. This presentation will review the CMS search strategy and prospects for SUSY discovery in the di-lepton final states.

European Physical Society Europhysics Conference on High Energy Physics July 16 - 22, 2009

Krakow, Poland

\footnotetext{
*Speaker.

†n behalf of the CMS collaboration.
} 
Introduction The Large Hadron Collider (LHC) at CERN and the CMS experiment [1] opens a new energy regime, $\sqrt{s}=14 \mathrm{TeV}$, offering a very exciting discovery potential for physics beyond the Standard Model, which has been extensively studied in the CMS Physics TDR [2]. In this presentation we show how the anticipated lower startup energy of $\sqrt{s}=10 \mathrm{TeV}$ will affect early Supersymmetry (SUSY) searches.

In the CMS experiment SUSY analyses are organized according to topologies, e.g. number of leptons and jets in the final state, which arise from SUSY cascades. The characteristics of these decays are large missing transverse energy (MET) from the lightest supersymmetric particle (LSP), high- $\mathrm{p}_{T}$ jets and lepton(s) in the final state. CMS has performed analyses with one or more leptons in the final state and in the following results of opposite sign di-lepton channels are summarized.

Opposite-sign di-lepton + jets + MET at $\sqrt{s}=10$ TeV Neutralino SUSY decays $\tilde{\chi}_{2}^{0} \rightarrow \tilde{\chi}_{1}^{0} \ell \ell$ lead to a sharp edge in the lepton pair mass distribution, which is illustrated in figure 1 (a) for all same-flavour, opposite-sign di-lepton pairs. The flavour symmetric background, dominated by SUSY decays, can be determined from data events with opposite-flavour opposite-sign leptons shown in figure 1 (b). With this method all backgrounds which produce uncorrelated leptons can be predicted such as $\mathrm{W}$, t-tbar, di-jet and di-boson events. After background subtraction the mass edge at about $50 \mathrm{GeV}$ is well visible in figure 1 (a) already with an integrated luminosity of 200 $\mathrm{pb}^{-1}$ at the low mass benchmark point LM0 [3].

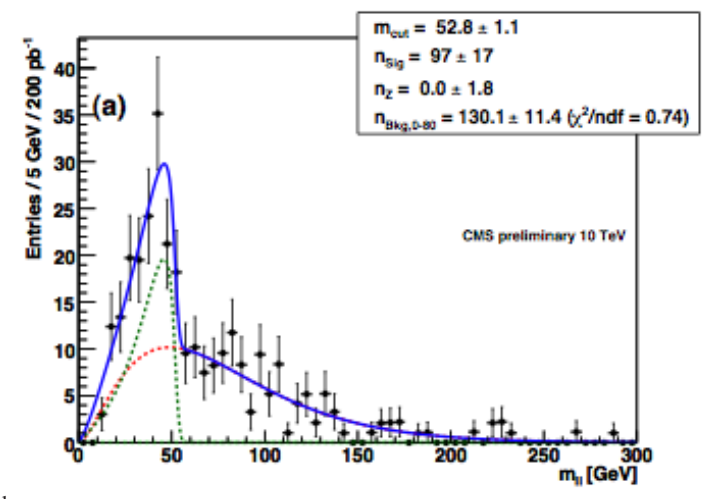

(a) Same-flavour lepton pair mass distriubtion.

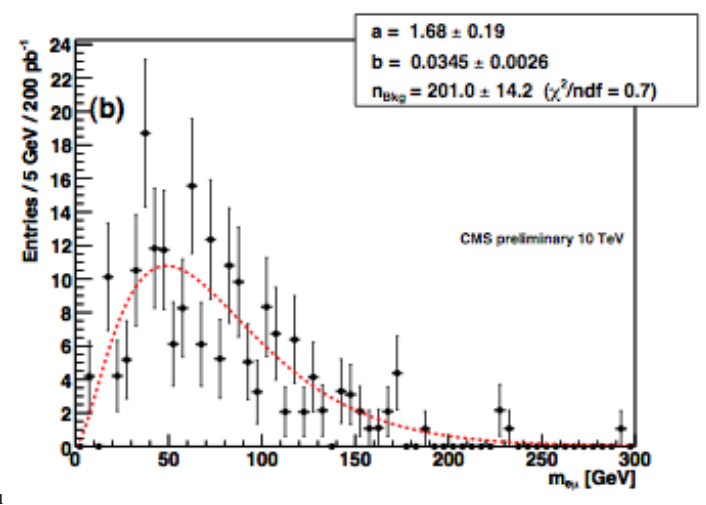

(b) Opposite-flavour lepton pair mass distribution.

Figure 1: The combined fit at LM0 for $200 \mathrm{pb}^{-1}$ is shown. The dashed lines identical in (a) and (b) represent the background and the peaking dashed line in (a) the SUSY signal. The full line shows the fit function, and the black points the MC events.

Using shape information, a significance of $5 \sigma$ can be achieved at the LM0 benchmark point with the first $200 \mathrm{pb}^{-1}$. With event counting alone, the same significance of $5 \sigma$ can be reached with $250 \mathrm{pb}^{-1}$. The corresponding numbers for the benchmark points LM1 and LM9 are also included in table 1. At the benchmark point LM0 the fit of the endpoint of the edge is possible with $200 \mathrm{pb}^{-1}$ (due to the large cross section of LM0), at LM1 and LM9 an integrated luminosity of 1 $\mathrm{fb}^{-1}$ is required. At LM0 the uncertainty for the fit of the endpoint is

$$
\Delta m_{\ell \ell}^{\max }= \pm 1.5 \text { (stat.) } \pm 0.9 \text { (syst.) } \mathrm{GeV} / \mathrm{c}^{2} .
$$


Table 1: Integrated luminosity for $5 \sigma$ significance using shape or pure event counting.

\begin{tabular}{|c||c|c|}
\hline & $\sigma_{\text {Shape }}$ & $\sigma_{\text {Count }}$ \\
\hline \hline LM0 & $200 \mathrm{pb}^{-1}$ & $250 \mathrm{pb}^{-1}$ \\
LM1 & $250 \mathrm{pb}^{-1}$ & $400 \mathrm{pb}^{-1}$ \\
LM9 & $350 \mathrm{pb}^{-1}$ & $600 \mathrm{pb}^{-1}$ \\
\hline
\end{tabular}

For LM0, the $\ell \ell$ mass resolution at $\sqrt{s}=10 \mathrm{TeV}$ and with $200 \mathrm{pb}^{-1}$ is slightly worse than for LM1 at design energy of $\sqrt{s}=14 \mathrm{TeV}$ and an integrated luminosity of $1 \mathrm{fb}^{-1}$, which was found to be [4]

$$
\begin{aligned}
& \Delta m_{e e}^{\max }= \pm 1.07 \text { (stat.) } \pm 0.36 \text { (syst.) GeV } / \mathrm{c}^{2} \\
& \Delta m_{\mu \mu}^{\max }= \pm 0.75 \text { (stat.) } \pm 0.18 \text { (syst.) GeV } / \mathrm{c}^{2} .
\end{aligned}
$$

Summary and Conclusion This paper covers SUSY studies with leptons in the final states with early data at $10 \mathrm{TeV}$ and compares them to the results at $14 \mathrm{TeV}$. At $10 \mathrm{TeV}$ the low mass benchmark point LM0 was analyzed, where a significant SUSY signal can be seen with an integrated luminosity of $200 \mathrm{pb}^{-1}$ of early data. The benchmark points LM1 and LM9 require slightly more integrated luminosity. At LM0 the di-lepton endpoint can be measured with $200 \mathrm{pb}^{-1}$, while at the points LM1 and LM9 $1 \mathrm{fb}^{-1}$ of data are needed.

\section{References}

[1] The CMS Collaboration, The CMS experiment at the CERN LHC, JINST 3:S08004, 2008.

[2] The CMS Collaboration, CMS Physics Technical Design Report, Volumne II: Physics Performance, J. of Phys. G: Nucl. Part. Phys. 34 (2007) 995-1579.

[3] The CMS Collaboration, Discovery potential and measurement of a dilepton mass edge in SUSY events at $\sqrt{s}=10 \mathrm{TeV}$, CMS PAS SUS-09-002, 2009.

[4] The CMS Collaboration, Dilepton + Jets + MET channel: Observation and Measurement $\tilde{\chi}_{2}^{0} \rightarrow \tilde{\chi}_{1}^{0} \ell \ell$, CMS PAS 2008/038, 2008. 